\title{
Altered migrating myoelectrical complex in an animal model of cholesterol gallstone disease: the effect of erythromycin
}

\author{
Q-W Xu, R B Scott, D T M Tan, E A Shaffer
}

\begin{abstract}
Background-The ground squirrel on a high cholesterol diet exhibits prolonged intestinal transit, a pathogenetic factor in cholesterol gallstone formation.

Aims-To examine the effect of a high cholesterol diet on the characteristics of the migrating myoelectrical complex (MMC) and the potential benefit of erythromycin.

Methods-Twenty four animals received either a trace (controls) or a $1 \%$ (high) cholesterol diet. After four weeks, five bipolar jejunal and terminal ileal electrodes were implanted. Seven days later, myoelectric activity was measured in conscious, fasted animals before and after treatment with erythromycin. Biliary lipid composition was assessed.
\end{abstract}

Results-Compared with controls, animals fed the high cholesterol diet exhibited a prolonged MMC cycle period (70 (6) versus 83 (3) minutes; $p<0.05$ ), whereas MMC migration velocity and the proportions of the MMC represented by phases I, II, and III were unchanged. Oral erythromycin significantly shortened the MMC cycle period in animals on the control and high cholesterol diet by $59 \%$ and $54 \%$ respectively, and increased the proportion of the cycle period occupied by phase III of the MMC in both dietary groups. Gall bladder bile became saturated with cholesterol and crystals developed in nine of 12 animals on the high cholesterol diet; controls had none.

Conclusion-Animals fed a high cholesterol diet had a prolonged MMC cycle period. This, along with diminished gall bladder motility, impairs the enterohepatic cycling of bile salts and reduces their hepatic secretion, contributing to the formation of abnormal bile. Erythromycin initiated more frequent cycling of the MMC. Its therapeutic value in cholesterol gallstone formation warrants further evaluation.

(Gut 1998;43:817-822)

Keywords: migrating myoelectrical complex; intestinal transit; cholesterol saturation index; gallstone disease; erythromycin

Dr E A Shaffer, Foothills Hospital, Department of Medicine, 140329 St NW, Calgary, Alberta, Canada T2N 2 T9.

Accepted for publication 4 June 1998

Cholesterol gallstone formation involves multiple hepatobiliary defects, ${ }^{1-6}$ and probably intestinal disorders as well..$^{7-10}$ Although the secretory defect in cholesterol gallstone disease initially represents a liver disorder, ${ }^{1-4}$ the gall bladder and intestine are not innocent bystanders. $^{3-12}$ By virtue of their active role in the enterohepatic circulation of bile salts, impaired gall bladder emptying and prolonged intestinal transit not only contribute to the "lithogenic" state, but also perpetuate it. ${ }^{246-8}$ In ground squirrels fed a high cholesterol diet, a model of cholesterol gallstone disease, ${ }^{13}{ }^{14}$ we have recently shown that prolonged intestinal transit, along with gall bladder stasis, is associated with a sluggish enterohepatic circulation of bile salts, an expanded deoxycholate pool, increased cholesterol saturation of bile, and the eventual formation of cholesterol crystals. ${ }^{14}$ These findings are consistent with reports in patients with cholesterol gallstones. ${ }^{7-10}$

Although the pathogenetic significance of prolonged intestinal transit in gallstone formation is gaining recognition both in gallstone patients ${ }^{7-10}$ and in experimental animals, ${ }^{14}$ little is known about the mechanisms that underlie this motility defect. ${ }^{410}{ }^{14}$ As the migrating myoelectrical complex (MMC) governs fasting intestinal transit ${ }^{15}{ }^{16}$ and thus the enteric limb of bile salt cycling during fasting, ${ }^{17-20}$ a time when supersaturated bile most frequently forms, ${ }^{2-4} 1920$ we studied the characteristics of the MMC in ground squirrels on a trace (control) and a $1 \%$ (high) cholesterol diet. In addition, if a defective MMC pattern was observed on feeding a high cholesterol diet, we sought to test whether erythromycin, a prokinetic agent, ${ }^{21-24}$ could stimulate increased frequency of the MMC and reverse the motility disorder in this model.

\section{Materials and methods}

EXPERIMENTAL ANIMALS

The University of Calgary Animal Care Committee approved this research protocol, which conforms to the guidelines of the Canadian Council on Animal Care. Richardson ground squirrels (Spermophilus richardsoni), with an average body weight of $300 \mathrm{~g}$, were trapped wild near Calgary. They were caged individually in thermoregulated rooms on a 12 hour/12 hour day/night light cycle and allowed free access to a standard chow diet and water for a minimum of four weeks. Twenty four animals were randomly divided into two groups (12 in each) and maintained throughout the duration (five weeks) of the experiment on either a control (chow) diet with trace cholesterol content
(0.027\%, United States Biochemical, Cleveland, Ohio) or a high cholesterol diet (United States Biochemical) comprising an identical chow, enriched with $1 \%$ cholesterol by weight. 
SURGICAL PREPARATION

After four weeks on their respective diets and following an 18 hour fast, animals were surgically prepared under general anaesthesia with halothane (Ayerst Laboratories, Montreal, Canada). Under sterile conditions, a plastic cannula bearing the electrode assembly (five pairs of Teflon coated, multistranded, stainless steel electrodes) was tunnelled subcutaneously from an interscapular exit site to the anterior abdominal wall and brought into the peritoneal cavity through a stab incision. ${ }^{25}$ The first three pairs of bipolar electrodes were fixed in the muscular layer (serosal side) of the jejunum at $10 \mathrm{~cm}$ intervals, with the first being 2.5 $\mathrm{cm}$ distal to the ligament of Treitz. The last two pairs were attached at $-10 \mathrm{~cm}$ and $-20 \mathrm{~cm}$ proximal to the ileocaecal valve. Finally, a reference electrode was secured in the muscle of the anterior abdominal wall. ${ }^{25}$ Animals were given oral fluids 12 hours after surgery and after 24 hours were returned to their respective diets ad libitum until recordings of fasting intestinal myoelectrical activity were obtained.

EXPERIMENTAL PROTOCOL

The first objective was to determine whether chronic feeding of the $1 \%$ high cholesterol diet was associated with changes in the characteristics of the MMC in this animal model. The recordings of fasting intestinal myoelectrical activity were obtained at the beginning of the sixth week of a trace $(n=12)$ or a high cholesterol diet $(n=12)$ in conscious animals after an 18 hour fast. During the recording period, animals were contained in a small cage $(15 \times 15 \times 20 \mathrm{~cm})$ with no medication or other restraint. Fasting electrical activity was recorded continuously for six to eight hours to document at least three full MMC cycles in all animals. Following this, a subgroup of animals on both diets proceeded to the next stage of the study in which the acute effect of erythromycin on the MMCs was assessed. Six animals in each dietary group were randomly selected to receive liquid erythromycin stearate (Abbott Laboratories, Montreal, Canada) $2 \mathrm{mg} / \mathrm{kg}$ orally $^{21-24}$ in the 10 minute interval immediately after the completion of phase III of an MMC. Two additional full cycles of the MMC were then recorded and the cycle period of the posterythromycin MMCs was compared with that of those prior to erythromycin administration. In separate experiments, two animals in each dietary group were randomly selected to receive an equal volume of liquid placebo, the vehicle without erythromycin, to detect any potential influence of handling related to feeding of either erythromycin or the vehicle.

DETERMINATION OF CHOLESTEROL SATURATION INDEX AND CRYSTAL INCIDENCE

At the end of the myoelectric recording, all animals were killed by cervical dislocation. The abdomen was opened, and bile was aspirated from the gall bladder using a sterile needle and syringe. One drop was immediately examined by polarised light microscopy for cholesterol crystals, identified as birefringent, notched rhomboidal plates ${ }^{14}$; the remainder was stored at $-70^{\circ} \mathrm{C}$ for later analysis. Gall bladder bile samples were analysed for total bile salts using the 3- $\alpha$-hydroxysteroid dehydrogenase assay (Worthington Biochemical Company, Freehold, New Jersey), for phospholipid by an enzymatic assay for choline (Boehringer Mannheim, Mannheim, Germany) and for cholesterol by an enzymatic diagnostic kit (Ames Division, Miles Laboratories, Slough, UK). ${ }^{14}$ Serum cholesterol concentrations were similarly determined by this Ames cholesterol kit. The cholesterol saturation index (CSI) was determined using Carey's critical tables for cholesterol solubility limits, based on total lipid concentrations and calculated using a computer program. ${ }^{1426}$

RECORDING AND ANALYSIS OF INTERDIGESTIVE INTESTINAL MYOELECTRICAL ACTIVITY

The bipolar electrodes were connected to the bioelectrical amplifier (model 8811A; HewlettPackard, Palo Alto, California) with lower and upper cut off frequencies of 0.05 and $300 \mathrm{~Hz}$, respectively). ${ }^{25}$ The output signals were recorded simultaneously on an eight channel chart recorder (model 7858A; HewlettPackard) and an eight channel FM tape recorder (model 3968A; Hewlett-Packard) for later replay and analysis. An oscilloscope was employed to set the spike processor window such that only electrical spiking activity with amplitude above the variation in slow wave potential was recorded..$^{25}$

The myoelectrical activities of the jejunum and terminal ileum were analysed by visual inspection of the original records. Fasting gastrointestinal electrical activity in the ground squirrel was characterised by the presence of slow waves with a superimposed pattern of recurrent spike burst activity which was cyclical in character with well defined phase I, II, and III activity typical of the migrating myoelectrical complex (MMC). ${ }^{15}{ }^{16} 25$ Phase III of the MMC was clearly identified as an aborally propagating band of intense electrical spike activity on every slow wave. It was preceded by a period of irregular electrical spike activity (phase II), and followed by a period of absent electrical spike activity (phase I). As phase III (the period of intensive myoelectrical spike activity) is the most distinctive and recognisable of the three phases, the cycle period was defined as the time interval between the end of two successive bursts of phase III activity. The propagation direction and velocity of the MMCs were determined by assessing the time and direction of passage of the phases of the MMC past bipolar electrodes 1-3 (the proximal jejunum) and 4-5 (the distal ileum). ${ }^{25}$

STATISTICS

Data are expressed as mean (SE). Comparisons between two groups were made using the paired and unpaired Student's $t$ test. Analysis of variance was utilised to assess the contribution of diet, erythromycin, and group to the duration of the MMC cycle. All calculations were performed using the computer program SYSTAT (SYSTAT Inc., Evanston, Illinois). Sta- 


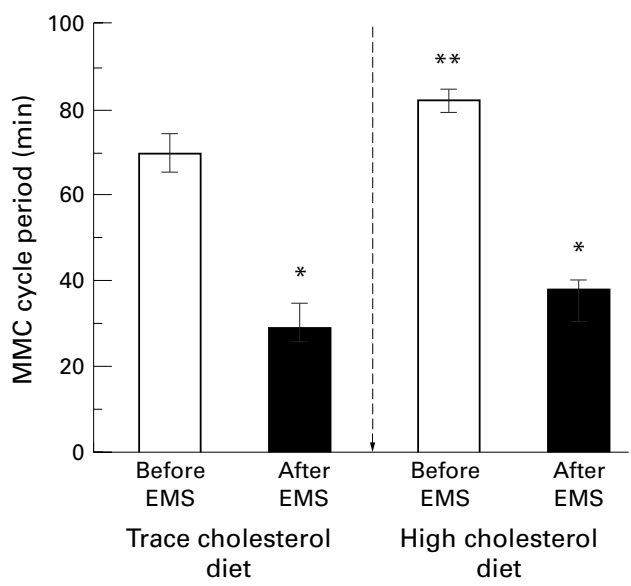

Figure 1 Effect of erythromycin on the MMC cycle period. EMS, erythromycin. ${ }^{\star} p<0.05$ versus controls; ${ }_{\star \star}^{*} p<0.05$ versus trace diet without erythromycin.

tistical values reaching probabilities of $p<0.05$ were considered significant.

\section{Results}

EFFECT OF THE HIGH CHOLESTEROL DIET ON THE CHARACTERISTICS OF THE ANIMAL

All animals tolerated the $1 \%$ high cholesterol diet and the surgical procedure well. They were healthy as assessed by their normal weight gain, shining coat, and normal activity. There were no significant differences in body weights (trace cholesterol, 457 (22) g versus high cholesterol, 498 (32) g; NS) between the two animal groups. Animals consuming the high cholesterol diet had a significant increase in their serum cholesterol concentrations (trace cholesterol, 5.2 (1.1) $\mathrm{mmol} / 1$ versus high cholesterol, 14.8 (3.2) $\mathrm{mmol} / \mathrm{l} ; \mathrm{p}<0.05)$. Compared with those on the trace cholesterol diet, animals on the high cholesterol diet exhibited no distinguishable histological alterations of the liver or small intestinal mucosa as assessed by conventional light microscopic examination of tissue sections.

EFFECT OF THE HIGH CHOLESTEROL DIET ON INTERDIGESTIVE INTESTINAL MYOELECTRICAL ACTIVITY

Fasting ground squirrels on both the trace and high cholesterol diets exhibited a characteristic cyclic pattern of intestinal myoelectrical activity typical of the MMC. This comprised three phases: quiescence (phase I), irregular (phase II), and intense, continuous, burst of myoelectrical spike activity (phase III). The MMCs migrated through the proximal jejunum to the terminal ileum. Compared with controls (trace cholesterol diet), the ground squirrels on the high cholesterol diet had a significantly prolonged MMC cycle period (trace cholesterol diet, 70 (6) minutes versus high cholesterol diet, 83 (3) minutes $(\mathrm{p}<0.05)$, showing a diminished frequency of the interdigestive MMC. The propagation velocity of the MMC did not change significantly (trace cholesterol diet, $3.5(0.6) \mathrm{cm} / \mathrm{min}$ versus high cholesterol diet, $2.8(0.6) \mathrm{cm} / \mathrm{min}$; NS). The proportion of the MMC cycle period representing each phase remained unchanged on the high versus trace cholesterol diet (phase I-trace cholesterol diet, 12.5 (4.0)\% versus high cholesterol diet, 14.1 (1.4)\%; phase II-trace cholesterol diet, $80.6(8.8) \%$ versus high cholesterol diet, 78.7 (1.3) $\%$; phase III-trace cholesterol diet, 7.0 $(0.9) \%$ versus high cholesterol diet, 7.1 (0.5)\%; all NS).

EFFECT OF ERYTHROMYCIN ON THE INTERDIGESTIVE INTESTINAL MYOELECTRICAL ACTIVITY

Oral administration of erythromycin stearate (2 $\mathrm{mg} / \mathrm{kg}$ orally) significantly shortened the cycle period of the next two complete cycles of the MMC by $59 \%$ and $54 \%$ in the trace and high cholesterol dietary groups, respectively (fig $1 ; \mathrm{p}<0.05$ ), whereas the administration of an equal volume of liquid placebo (the vehicle for the erythromycin stearate) had no effect on the MMC cycle period in either group (data not shown). The addition of erythromycin had no significant effect on propagation velocity in either dietary group (table 1). Erythromycin significantly increased the proportion of the MMC cycle period represented by phase III activity whereas it decreased the proportion of the MMC cycle period represented by phase II activity in both dietary groups (a fourfold and twofold increase in trace and high cholesterol diet groups, respectively; table $1 ; \mathrm{p}<0.05$ ).

Analysis of variance was utilised to assess the contribution of diet, diet plus erythromycin, and diet plus erythromycin plus group to the duration of the MMC cycle. Prior to treatment with erythromycin, there was a significant $(\mathrm{p}<0.05)$ effect of diet on MMC cycle period (it was prolonged on the high cholesterol diet). However, when the effect on MMC cycle period of diet plus treatment with erythromycin was evaluated, the only significant effect $(p<0.01)$ was from treatment with erythromycin. This can be explained by the fact that before treatment with erythromycin, MMC cycle period was significantly prolonged on the high versus trace cholesterol diet (83 (3) versus 70 (6) minutes; $\mathrm{p}<0.05$ ), However, treatment with erythromycin greatly reduced MMC cycle duration in both treatment groups to compara-

Table 1 Effect of erythromycin on interdigestive intestinal myoelectrical activity

\begin{tabular}{llllll}
\hline & \multirow{2}{*}{ Trace cholesterol diet } & & & \multirow{2}{*}{ High cholesterol diet } \\
\cline { 2 - 3 } \cline { 5 - 6 } & Before erythromycin & After erythromycin & & Before erythromycin & After erythromycin \\
\hline Phase I (\% MMC cycle period) & $12.5(4.0)$ & $35.9(11.6)$ & & $14.1(1.4)$ & $24.2(7.8)$ \\
Phase II (\% MMC cycle period) & $80.6(4.4)$ & $43.7(12.3)^{\star}$ & & $78.7(1.3)$ & $58.3(8.1)^{\star}$ \\
Phase III (\% MMC cycle period) & $7.0(0.9)$ & $21.1(4.2)^{\star}$ & & $7.1(0.5)$ & $15.2(1.7)^{\star}$ \\
Velocity & $3.5(0.6)$ & $2.9(0.7)$ & & $2.8(0.6)$ & $3.9(0.5)$ \\
\hline
\end{tabular}

Results are expressed as mean (SE).

${ }^{\star} \mathrm{p}<0.05 v$ respective controls. 
ble values that were not affected by dietary treatment (52 (7) minutes in the trace cholesterol versus 47 (6) minutes in the high cholesterol group treated with erythromycin; $\mathrm{p}<0.01)$.

EFFECT OF THE HIGH CHOLESTEROL DIET ON CHOLESTEROL SATURATION INDEX AND

CHOLESTEROL CRYSTAL INCIDENCE

The $1 \%$ high cholesterol diet notably increased the cholesterol saturation in gall bladder bile (trace cholesterol diet, $0.58(0.06)$ versus high cholesterol diet, $1.2(0.08) ; \mathrm{p}<0.05)$. This was largely due to a higher molar percentage of cholesterol (trace cholesterol diet, $3.0(0.4) \%$ versus high cholesterol diet, $7.1 \quad(0.5) \%$; $\mathrm{p}<0.05)$ and a lower molar percentage of bile salts (trace cholesterol diet, $86.2(1.1) \%$ versus high cholesterol diet, $78.1(0.6) \%$; $<<0.05)$.

Macroscopically, fresh gall bladder bile samples from animals on the trace cholesterol diet were clear without visible precipitates. In animals on the $1 \%$ high cholesterol diet, all bile samples were turbid, filled with sand like precipitates. Under polarising microscopic examination ( $\mathrm{n}=12$ in each group), no cholesterol crystals were found in the initial (native) gall bladder bile of any animal on the trace cholesterol (control) diet. In contrast, nine of 12 cholesterol fed animals had solid cholesterol crystals and three had macroscopic cholesterol stones.

\section{Discussion}

The present study shows that when ground squirrels consume a $1 \%$ high cholesterol diet, a model of cholesterol gallstone formation, ${ }^{13} 1427$ their MMC cycle periods become significantly prolonged. This reduction in MMC cycle frequency in animals on the high cholesterol diet correlates with a previously shown delay in small intestinal transit in this model, ${ }^{14}$ an effect that contributes to the formation of bile saturated with cholesterol and development of cholesterol crystals. ${ }^{7-10}$ Furthermore, the findings show that erythromycin significantly shortens the MMC cycle period and notably increases the proportion of the cycle period occupied by propulsive phase III activity of the MMC in both dietary groups - an observation with therapeutic potential. ${ }^{24}$

The hepatic secretion of bile supersaturated with cholesterol, the first stage of cholesterol gallstone formation, is primarily a metabolic disorder of the liver, being driven by a combination of diet and genetic predisposition. ${ }^{1-4}$ This "abnormal" bile seems to disrupt the normal motor function of organs downstream (the gall bladder and the small intestine) through as yet unknown mechanisms. ${ }^{413} 1428$ The ensuing gall bladder stasis and prolonged intestinal transit impede the enterohepatic cycling of bile salts with a resultant reduction in their hepatic secretion $^{2} 8^{14}{ }^{18-20}$ and a simultaneous increase in deoxycholate pool size..$^{7-101429}$ These interdependent events create a vicious circle that promotes the secretion of bile saturated with cholesterol $^{4}$ and the subsequent formation of gallstones, both in patients with cholesterol gallstones, ${ }^{7-10}$ and in experimental animal models. ${ }^{14}$

Although the concept is not new, ${ }^{30}$ the pathogenic role of prolonged intestinal transit in gallstone formation has been largely ignored until recently. ${ }^{10}$ There are several mechanisms which could be invoked as a cause of prolonged intestinal transit in these experiments. In the human, the fibre content of the diet is known to be an important determinant of intestinal transit time. In this study, the diets of the trace and high cholesterol diet treatment groups were identical, including the fibre content, and differed only in the amount of cholesterol ingested. Thus, a difference in fibre intake would not explain the prolonged intestinal transit time in the ground squirrel model of cholesterol gallstone formation. Another potential mechanism which might explain the prolonged intestinal transit time in this model relates to the fasting pattern of gastrointestinal motility. Under fasting conditions, the upper gastrointestinal tract exhibits a cyclic pattern of motor activity, namely the MMC. ${ }^{16}$ The MMC propagates aborally as far as the ileocaecal junction. ${ }^{15}{ }^{16}$ Its cyclic appearance in the duodenum is associated with partial emptying of the gall bladder and the pulsatile delivery of bile into the duodenum. ${ }^{17-19}$ It is followed by the aboral propulsion of bile salts to their site of active reabsorption in the distal ileum, ${ }^{19}{ }^{31}$ resulting in augmented return of bile salts to the liver for their resecretion. ${ }^{17-20}$ Thus, the magnitude of hepatic bile salt secretion during fasting is a function of enterohepatic cycling, powered by intermittent emptying of the gall bladder and the propulsive activity of the small intestine, ${ }^{17-19}$ all of which is determined mainly by the periodicity of the MMC. ${ }^{15-19}$ The present study shows that, during fasting, animals on a high cholesterol diet exhibit a significant prolongation of their MMC cycle period, compared with controls. The functional consequence of this abnormality is a reduction in the number of MMC cycles, less frequent partial emptying of the gall bladder, and less frequent propulsion of luminal bile salts to their distal site of absorption during a prolonged fast (overnight). ${ }^{15-203132}$ These alterations in fasting intestinal motility may, in part, explain the previously observed delay in small intestinal transit in animals on the high cholesterol diet.

Although there is no information about how the pathophysiology of gallstone formation alters the periodicity of the MMC, it is conceivable that the changes in MMC cycle frequency in the present study may have resulted from the high cholesterol diet. ${ }^{1428} 33$ Under normal conditions, about $50-60 \%$ of total dietary cholesterol is absorbed in the upper portion of the intestine ${ }^{34}$; some eventually reaches the terminal ileum. The $1 \%$ high cholesterol diet represents a 40 -fold increase in cholesterol content, compared with the control diet with only $0.027 \%$ cholesterol by weight. We speculate that the entire small intestine is exposed to unusually high concentrations of cholesterol, as a consequence of an overload of 
dietary and biliary cholesterol, which exceeds the absorptive capacity of the proximal small intestine. ${ }^{14} 3134$ Conceivably, the excess cholesterol reaches and is incorporated into the neuronal and/or smooth muscle membranes of the small intestine. ${ }^{4131428}$ This modifies membrane fluidity ${ }^{35}$ and may thereby affect function of the membrane regulatory proteins, such as the receptors and ion channels ${ }^{48} 8^{33} 3^{35-38}$ that control electrical excitability and influence the periodicity of events like the MMC. ${ }^{15}{ }^{16} \mathrm{Al}-$ though the present findings suggest a correlation between increased luminal free cholesterol and altered MMC in the ground squirrel, the situation in humans may differ as patients with cholesterol gallstones do not necessarily have high concentrations of cholesterol either in the intestinal lumen or in the blood. ${ }^{34}$ They do however secrete excessive biliary cholesterol into the small intestine. ${ }^{1}{ }^{2} 39$

The prolonged MMC cycle period and the delayed intestinal transit in the ground squirrel model of cholesterol cholelithiasis coincide with both increased deoxycholate content and hydrophobicity of the total bile salt pool. ${ }^{14}$ Hydrophobic bile salts such as deoxycholate are more cytotoxic. ${ }^{40-42}$ By forming water soluble complexes with the phospholipid components of the membrane, even at concentrations well below their critical micellar concentrations, ${ }^{43}$ these hydrophobic bile salts might also be capable of altering the structurefunction of neuronal membranes, ${ }^{40-46}$ which regulate cyclic activity within the enteric nervous system. ${ }^{15}{ }^{16}$ This might then lead to the observed reduction in MMC cycle frequency and the prolonged intestinal transit. ${ }^{15} 16$ Additional experiments, however, are necessary to clarify the true mechanism of delayed intestinal transit in this model of cholesterol gallstone formation.

Erythromycin, a 13 member macrolide compound, has a powerful prokinetic effect on both the biliary and gastrointestinal tracts, ${ }^{21-24}$ probably by binding to motilin receptors and acting as its agonist. ${ }^{22}$ Like the regulatory peptide motilin, ${ }^{115} 16$ erythromycin induces phase III activity that starts in the upper gastrointestinal tract and migrates through the small intestine..$^{21-23}$ In the present study, oral administration of erythromycin significantly shortened the MMC cycle period in ground squirrels from both dietary regimes and increased the proportion of the MMC cycle occupied by phase III, the intense and propulsive activity front of the MMC. ${ }^{15}{ }^{16}$ The physiological consequence of these changes was an accelerated intestinal transit and more rapid enterohepatic cycling of bile salts. ${ }^{17-20}$ Such accelerated intestinal transit and more rapid enterohepatic cycling of bile salts has the potential to increase bile salt secretion, improve cholesterol solubility, and reduce crystal development. The mechanism of the accelerated transit relates to an increased cycle frequency of the MMC. In the fasting state, partial gall bladder emptying occurs cyclically in association with the MMC; peak duodenal bile delivery occurs at $70 \%$ of the duodenal cycle period. ${ }^{18}$ The transport of duodenal bile acids to their distal site of ileal absorption and thence to the liver is pulsatile rather than continuous and also determined by the MMC. ${ }^{47}$ Maximum uptake from the distal site of reabsorption occurs when phase III of the MMC propels the intraluminal bile acid pool to its site of absorption in the distal small bowel. Bile acids delivered into the duodenum just prior to duodenal phase III are propelled aborally by the activity front of phase III activity to their distal sites of absorption. The "housekeeping" action of the MMC is very efficient and $88 \%$ of bile acids delivered into the duodenum are propelled aborally by the activity front of the first passing MMC and reabsorbed. Animals with more frequent MMCs will on average propel luminal contents more distally than animals with less frequent MMCs. This will occur even though the velocity of propagation of the individual MMCs is identical. Thus, an altered MMC cycle frequency would, in part, explain the previously observed delay in small intestinal transit in animals on the high cholesterol diet. ${ }^{14}$ What is actually accounting for the erythromycin effect, though, is an increased cycling frequency, not an increase in propagation velocity of the individual MMCs.

Erythromycin induced improvement in intestinal and gall bladder motor function may offer a potential therapeutic intervention that might alleviate the clinical consequences of bile saturated with cholesterol. ${ }^{12}{ }^{24}$ 48-50 In fact, recent preliminary findings of a trial of four weeks treatment with erythromycin in the same animal model support this hypothesis. ${ }^{51}$

In conclusion, we have shown that ground squirrels fed a high cholesterol diet exhibit a significant prolongation of their MMC cycle period. This may, in part, provide an explanation for the previously shown delay in aboral intestinal transit during fasting in this model, ${ }^{14}$ which along with diminished gall bladder motility impairs the enterohepatic cycling of bile salts and reduces their hepatic secretion. ${ }^{2} 814$ 17-20 48-50 The resulting sluggish cycling of bile salts may serve to expand the deoxycholate pool ${ }^{4-1014}$ and contribute to the formation of bile saturated with cholesterol. ${ }^{30}$ Lastly, the demonstration that erythromycin significantly increased MMC cycle frequency in the cholelithiasis group suggests that treatment with erythromycin may be of potential therapeutic value in prevention of cholesterol gallstone formation, ${ }^{24} 48-50$ a therapy warranting further evaluation.

\footnotetext{
1 Grundy SM, Metzger AL, Adler RD. Mechanisms of lithogenic bile formation in American Indian women with cholesterol gallstones. $\mathcal{F}$ Clin Invest 1972;51:3026-43.

2 Shaffer EA, Small DM. Biliary lipid secretion in cholesterol gallstone disease. The effect of cholecystectomy and obesity. F Clin Invest 1977;59:828-40.

3 Paumgartner G, Sauerbruch T. Gallstones: pathogenesis. Lancet 1991;338:1117-21.

4 Carey MC. Formation and growth of cholesterol gallstone: the new synthesis. In: Fromm H, Leuschner Y, eds. Bile acids-cholesterol gallstones (Falk symposium No. 84). Dordrecht: Kluwer, 1996:147-75.

5 Pomeranz IS, Shaffer EA. Abnormal gall bladder emptying in a subgroup of patients with gallstones. Gastroenterology

6 Jazrawi RP, Pazzi P, Petroni ML, et al. Postprandial gall bladder motor function: refilling and turnover of bile in health and in cholelithiasis. Gastroenterology 1995;109:58291 .
} 
7 Heaton KW, Emmett PM, Symes CL, et al. An explanation for gallstones in normal-weight women: slow intestinal

8 Shoda J, He B-F, Tanaka N, et al. Increase of deoxycholate in supersaturated bile of patients with cholesterol gallstone disease and its correlation with de novo synthesis of cholesterol and bile acids in liver, gall bladder emptying and smal intestinal transit. Hepatology 1995;21:1291-302.

9 Dowling RH, Hussaini SH, Murthy GM, et al. Gallstones during octreotide therapy. Metabolism 1992;41:22-33.

10 Dowling RH, Veysey MJ, Pereira SP, et al. Role of intestinal transit in the pathogenesis of gall bladder stones. Can $\mathcal{F}$ Gastroenterol 1997;11:57-64.

11 Stolk MFJ, van Erpecum KJ, Smout AJPM, et al. Motor cycles with phase III in antrum are associated with high motilin levels and prolonged gall bladder emptying. Am $\mathcal{F}$ Physiol 1993;264:G590-600.

12 O'Donnell LJD, Wilson P, Guest P, et al. Indomethacin and postprandial gall bladder emptying. Lancet 1992;339:269postprar 70 .

$13 \mathrm{Xu}$ QW, Shaffer EA. The potential site of impaired gall bladder contractility in an animal model of cholesterol gallstone disease. Gastroenterology 1996;110:251-7.

$14 \mathrm{Xu}$ QW, Scott RB, Tan D, et al. Slow intestinal transit: a motility disorder contributing to cholesterol gallstone formation in the ground squirrel. Hepatology 1996;23: 1664-72.

15 Szurzewski JH. Electrical basis of gastrointestinal motility. In: Johnson LR, ed. Physiology of the gastrointestinal tract. 2nd edn. New York: Raven Press, 1987:383-422.

16 Christensen J. Motility of the intestine. In: Sleisenger $\mathrm{MH}$ Fordtran JS, eds. Gastrointestinal disease, 5th edn. Philadelphia: Saunders, 1993:822-37.

17 Scott RB, Strasberg SM, El-Sharkawy TY, et al. Fasting canine biliary secretion and sphincter of Oddi. Gastroenterology 1984;87:793-804.

18 Scott RB, Eidt PB, Shaffer EA. Regulation of the fasting canine duodenal bile acid delivery by sphincter of Oddi and canine duodenal bile acid delivery by sphincter

19 Carey MC, Duane WC. Enterohepatic circulation. In: Arias IM et al, eds. The liver: biology and pathobiology. 3rd edn. New York: Raven Press, 1994:719-68.

20 Everson GT, Lawson MJ, McKinley C. Gall bladder and small intestinal regulation of biliary lipid secretion during intraduodenal infusion of standard stimuli. $\mathcal{F}$ Clin Invest 1983;71:596-603.

21 Itoh Z, Nakaya M, Suzuki T, et al. Erythromycin mimics Suztivity in the dog. Am f Physiol 1984;247:G688-94.

22 Peeters TL. Erythromycin and other macrolides as prokinetic agents. Gastroenterology 1993;88:1886-99.

23 Tomomasa T, Kuroume T, Arai H, et al. Erythromycin induces migrating motor complex in human gastrointestinal tract. Dig Dis Sci 1986;31:157-61.

24 Catnach SM, Fairclough PD, Trembach RC, et al. Effect of oral erythromycin on gall bladder motility in normal subjects and subjects with gallstones. Gastroenterology 1992;102:2071-6.

25 Scott RB, Diamant SC, Gall DG. Motility effect of intestinal anaphylaxis in the rat. Am $\mathcal{F}$ Physiol 1988;255. intestinal

26 Carey MC. Critical tables for calculating the cholesterol saturation of native bile. $\mathcal{F}$ Lipid Res 1978;19:945-55.

27 MacPherson BR, Pemsingh RS, Scott GW. Experimental cholelithiasis in the ground squirrel. Lab Invest 1987;56 138-45.

28 Yu P, Chen Q, Biancani P, Behar J. Membrane cholesterol alters gall bladder muscle contractility in prairie dogs. $A m \mathcal{F}$ Physiol 1996;271:G56-61.

29 Berr F, Stellaard F, Pratschke E, Paumgartner G. Effects of cholecystectomy on the kinetics of primary and secondary bile acids. $\mathcal{F}$ Clin Invest 1989;83:1541-50.
30 Marcus SN, Heaton KW. Deoxycholic acid and the pathogenesis of gallstones. Gut 1988;29:522-33.

31 Hofmann AF. Bile acids. In: Arias IM, et al, eds. The liver: biology and pathobiology. 2nd edn. New York: Raven Press, 1988:553-72.

32 Metzger AL, Adler R, Heymsfield S, et al. Diurnal variation in biliary lipid composition. Possible role in cholesterol gallstone formation. N Engl f Med 1973;288:333-6.

$33 \mathrm{Kim} \mathrm{JH}$, Klyachkin ML, Svendsen E, et al. Experimental hypercholesterolemia in rabbits induces cavernosal atherosclerosis with endothelial and smooth muscle cell dysfunction. F Urol 1994;151:198-205.

34 Kern F, Jr. Effect of dietary cholesterol on cholesterol and bile acid homeostasis in patients with cholesterol gallstones. F Clin Invest 1994;93:1186-94.

35 Chen Q, Yu P, Biancani P, Behar J. Excess membrane cholesterol alters membrane fluidity of gall bladder muscle cells [abstract]. Gastroenterology 1996;110:A451.

36 Broderick R, Bialecki R, Tulenko T. Cholesterol-induced changes in rabbit arterial smooth muscle sensitivity to adrenergic stimulation. Am $\mathcal{f}$ Physiol 1989;257:H170-8.

37 Bialecki RA, Tulenko TN. Excess membrane cholesterol alters calcium channels in arterial smooth muscle. Am $\mathcal{F}$ Physiol 1989;257:C306-14.

38 Cox RH, Tulenko TN. Altered contractile and ion channel function in rabbit portal vein with dietary atherosclerosis. Am 7 Physiol 1995; 268: H2522-30.

39 Nilsell K, Angelin B, Liljeqvist L. Biliary lipid output and bile acid kinetics in cholesterol gallstone disease. Evidence for an increased hepatic secretion of cholesterol in Swedish patients. Gastroenterology 1985;89:287-93.

40 Roda A, Grigolo B, Pellicciari R, et al. Structure-activity relationship studies on natural and synthetic bile acid analogs. Dig Dis Sci 1989;34(suppl):24-35S

41 Lillemoe KD, Kidder GW, Harmon JW, et al. Tauroursodeoxycholic acid is less damaging than taurochenodeoxycholic acid to the gastric and esophageal mucosa. Dig Dis Sci 1983;28:359-64.

42 Schmucker DL, Ohta M, Kanai S, et al. Hepatic injury induced by bile salts: correlation between biochemical and morphological events. Hepatology 1990;12:1216-21.

43 Shoemaker DG, Nichols JW. Hydrophobic interaction of ysophospholipids and bile salts at submicellar concentrations. Biochemistry 1990;29:5837-42.

44 Heuman DM, Bajaj R. Ursodeoxycholate conjugates protect against disruption of cholesterol-rich membranes by bile salts. Gastroenterology 1994;106:1333-41.

$45 \mathrm{Xu}$ Q-W, Shaffer EA. The influence of bile salts on small intestinal motility in the guinea pig in vitro. Gastroenterology 1992;103:29-35.

$46 \mathrm{Xu}$ Q-W, Shaffer EA. Inhibitory effect of bile salts on gall bladder smooth muscle contractility in the guinea pig in vitro. Gastroenterology 1997;112:1699-706.

47 Scott RB, Strasberg SM, El-Sharkawy TY, et al. Regulation of the fasting enterohepatic circulation of bile acids by the migrating myoelectric complex in dogs. F Clin Invest 1983; 71:644-54.

48 Roslyn JJ, DenBesten L, Pitt H, et al. Effect of cholecystokinin on gall bladder stasis and cholesterol gallstone formation. F Surg Res 1981;30:200-4.

49 Doty JE, Pitt HA, Porter-Fink V, et al. Cholecystokinin prophylaxis of parenteral nutrition-induced gall bladder prophylaxis of parenteral nutrition-
disease. Ann Surg 1985;201:76-80.

$50 \mathrm{Xu}$ Q-W, Shaffer EA. Cisapride improves gall bladder contractility and bile lipid composition in an animal model of gallstone disease. Gastroenterology 1993;105:1184-91.

$51 \mathrm{Xu}$ QW, Scott RB, Tan D, et al. Erythromycin as a prokinetic agent in an animal model of cholesterol gallstone disease [abstract]. Gastroenterology 1995;108:A441. 